\title{
Dissecting parkinsonism: cognitive and gait disturbances
}

\author{
Philip W. Tipton \\ Department of Neurology, Mayo Clinic, Jacksonville, Florida, United States
}

\begin{abstract}
Parkinsonism is usually designated a movement disorder. However, cognitive impairment comprises a major part of many parkinsonian syndromes, and inversely correlates with quality of life. Parkinsonian features are largely attributed to subcortical dopaminergic dysfunction, although other brain regions and neurotransmitters also contribute. This is illustrated by phenotypic differences among different parkinsonian syndromes. Slowed processing speed and executive dysfunction are generally expected in parkinsonian patients, but additional cognitive features can suggest specific pathological substrates, e.g. apraxia in corticobasal degeneration. Similarly, motor symptoms generally include combinations of bradykinesia, rigidity, rest tremor, and postural instability, although nuanced differences and associated clinical features often help differentiate between parkinsonian syndromes. Human gait requires complex synchronisation at every level of the nervous system, yet occurs with minimal conscious effort on behalf of the walker. Deviations from the normal gait pattern can result from otherwise unnoticeable insults to the body, both intrinsic and extrinsic to the nervous system. Gait is almost always abnormal in parkinsonism, ranging from subtle arm swing asymmetry to discrete episodes of gait freezing. Moreover, one's cognitive state can directly affect one's quality of gait. The notion that a gait profile could in fact be a disease-specific biomarker is an area of great research interest. This review focuses on the manifestations of, and correlations between, cognitive and gait impairment in common parkinsonian conditions, and provides guidance for a clinical approach to assessing them.
\end{abstract}

Key words: parkinsonism, cognition, gait, falls

(Neurol Neurochir Pol 2021; 55 (6): 513-524)

\section{Introduction}

Parkinsonism broadly describes a constellation of symptoms including bradykinesia, rigidity, rest tremor, postural instability, flexed posture, and freezing. These symptoms are largely the result of dopaminergic dysfunction with legion manifestations of varying degrees of severity, ranging from a classical rest tremor to subtle hypomimia. Individuals with parkinsonism almost always have gait disturbances that can vary depending on the underlying cause. Recognising these nuanced differences helps the clinician to make an accurate and prompt diagnosis, which can have prognostic and treatment implications.

Parkinsonism is often accompanied by non-motor symptoms. For instance, Parkinson's Disease (PD) manifests with many non-motor symptoms that can be equally disabling as, or even more disabling than, motor symptoms [1]. Cognitive impairment is one such non-motor symptom that is common in PD and associated with a reduced quality of life (QoL) [2]. Cognitive impairment frequently accompanies other forms of parkinsonism in various ways, underlining the importance of acquiring a detailed cognitive profile in order to assist in diagnosis.

This review describes the various gait and cognitive manifestations of the most common parkinsonian conditions, addresses connections between gait and cognitive impairment, and offers guidance for assessing cognition and gait.

\section{Cognitive impairment in parkinsonism}

\section{Parkinson's Disease}

PD is classically labelled as a movement disorder; however, non-motor symptoms constitute a large part of the phenotype and result in significant disability [1]. Cognitive

Address for correspondence: Philip W. Tipton, Department of Neurology, Mayo Clinic, 4500 San Pablo Rd., Jacksonville, FL 32224, USA;

e-mail: tipton.philip@mayo.edu

Received: 10.09.2021 Accepted: 5.11.2021 Early publication date: 24.11.2021

This article is available in open access under Creative Common Attribution-Non-Commercial-No Derivatives 4.0 International (CC BY-NC-ND 4.0) license, allowing to download articles and share them with others as long as they credit the authors and the publisher, but without permission to change them in any way or use them commercially. 
Table 1. Typical cognitive features of parkinsonian disorders

\begin{tabular}{|c|c|c|c|c|c|c|c|c|c|c|c|c|c|c|}
\hline & \multirow[t]{2}{*}{ PD } & \multirow[t]{2}{*}{ DLB } & \multicolumn{2}{|c|}{ MSA } & \multicolumn{6}{|c|}{ PSP } & \multirow[t]{2}{*}{ CBD } & \multirow[t]{2}{*}{ NPH } & \multirow{2}{*}{$\begin{array}{l}\text { Vasc- } \\
\text {-Park }\end{array}$} & \multirow{2}{*}{$\begin{array}{l}\text { Med- } \\
\text {-Park }\end{array}$} \\
\hline & & & $\mathbf{P}$ & C & RS & $\mathbf{P}$ & C & CBS & SL & FTD & & & & \\
\hline \multicolumn{15}{|l|}{ Cognitive domain } \\
\hline Processing speed & $+^{*}$ & + & + & + & + & + & + & + & + & + & + & + & \pm & \pm \\
\hline Executive function & $+^{*}$ & + & + & $t^{\#}$ & $t^{5}$ & + & + & + & + & + & + & + & \pm & \pm \\
\hline Memory & - & + & - & - & - & - & - & - & - & - & - & + & \pm & - \\
\hline Verbal fluency & - & - & + & - & $t^{\&}$ & - & - & $+^{+}$ & \pm & \pm & $t^{+}$ & \pm & \pm & - \\
\hline Naming & - & - & - & - & - & - & - & \pm & \pm & - & - & - & \pm & - \\
\hline Visuospatial skills & + & + & + & + & - & - & - & + & - & - & + & - & \pm & - \\
\hline \multicolumn{15}{|l|}{ Behavioural features } \\
\hline Visual hallucinations & + & + & \pm & - & - & - & - & - & - & - & - & - & - & + \\
\hline Auditory hallucinations & - & - & - & - & - & - & - & - & - & - & - & - & - & + \\
\hline Limb apraxia & - & - & - & - & - & - & - & + & - & - & + & - & \pm & - \\
\hline Apraxia of speech & - & - & - & - & - & - & - & \pm & \pm & - & \pm & - & - & - \\
\hline Disinhibition & - & - & - & - & - & - & - & - & - & - & \pm & - & \pm & \pm \\
\hline Pseudobulbar affect & + & + & + & + & + & + & + & \pm & \pm & + & \pm & - & + & - \\
\hline Apathy & + & - & - & - & $t^{*}$ & $t^{*}$ & - & - & - & - & \pm & + & \pm & \pm \\
\hline
\end{tabular}

+ impaired; - unimpaired; \pm variably impaired; *Relatively early in disease course compared to other cognitive impairments in PD. "Especially impaired attention [19]; ${ }^{5}$ Especially spatial planning and set shifting [30]; ${ }^{8}$ Early phonological verbal fluency deficits [35]; ${ }^{*}$ Dependent on which brain hemisphere is predominantly affected; ${ }^{*} A$ pathy is more prevalent in PSP-P compared to PSP-RS [35]

CBD - corticobasal degeneration; DLB - dementia with Lewy bodies; Med-Park - medication-induced parkinsonism; MSA - multiple system atrophy; MSA-C - MSA cerebellar subtype; MSA-P — MSA parkinsonian subtype; NPH — normal pressure hydrocephalus; PD — Parkinson's Disease; PSP — progressive supranuclear palsy; PSP-CBS — PSP corticobasal syndrome subtype; PSP-FTD — PSP frontotemporal dementia subtype; PSP-P — PSP with predominant parkinsonism; PSP-RS — PSP Richardson's Syndrome; PSP-SL — PSP with predominant speech/language disorder; Vasc-Park — vascular parkinsonism

impairment is common in $\mathrm{PD}$, correlates to lower QoL, and predicts caregiver 'burnout' [2]. Heterogeneity in the cognitive trajectory of patients with PD suggests that there are multiple contributory factors. Cognitive impairment has typically been considered a late disease feature with a nonlinear rate of change characterised by gradual decline before accelerating to more rapid decline approximately 13 years into the disease [3]. This model has been challenged by studies showing that mild cognitive impairment (MCI) can occur early in the disease and is identified in $18.9 \%$ of drug-naive PD patients with an average disease duration of 2.4 years [4]. Patients with PD-MCI can remain stable for many years, or even revert to normal cognition [5]. However, PD-MCI significantly increases one's risk of progressing to PD-dementia (PD-D) [6]. Patients with $\mathrm{PD}$ are nearly six times more likely to become demented than those without PD, and $80 \%$ of patients will have developed dementia 15-20 years into the disease course $[7,8]$.

Non-amnestic single-domain impairment is the most common subtype of PD-MCI, but there is variability among patients [9]. Williams-Gray et al. identified two distinct cognitive profiles in early PD [10]. They found that a frontostriatal dysexecutive profile was common in early disease and less likely to progress to dementia, as opposed to a posterior cortical profile that primarily affected visuospatial and memory domains and was more likely to progress to dementia (Tab. 1). Frontal executive impairments may result from nonuniform dopaminergic loss in 'fronto-striatal loops' which are relatively spared dorsally compared to the more ventral loops that are implicated in bradykinesia and rigidity [11]. The posterior cortical cognitive profile may correlate with increased burden of cortical Lewy bodies and result from cholinergic systems dysfunction rather than disrupted dopamine signalling [10]. A recent study was not powered to valid this hypothesis, but it did show more severe cortical Lewy body pathology in non-amnestic versus amnestic PD-MCI [12].

\section{Atypical parkinsonian syndromes}

\section{Dementia with Lewy bodies (DLB)}

When evaluating a patient with parkinsonism, one must monitor for 'red flags' that suggest an atypical parkinsonian neurodegenerative disease. The timing and character of cognitive symptoms provide clinicians with useful insights into the underlying pathology. The DLB cognitive profile is typically characterised by deficits in attention, executive function, and visual processing more than in memory or naming (Tab. 1) [13]. Notable core cognitive/behavioural diagnostic features of DLB include fluctuating attention/alertness and recurrent well-formed visual hallucinations [13]. Like PD, dementia with Lewy bodies (DLB) results from abnormal a-synuclein deposition. Despite the shared designation of Lewy body disease (LBD), their significant clinical differences have implications for treatment and prognosis. Applying the 'DLB 1-year rule' (cognitive impairment before or within 12 months of motor symptom onset) provides a quick means of distinguishing between these two diseases, although this 
concept is based on an outdated understanding of the PD cognitive profile.

We now know that cognitive impairment is common early in PD and that cognitive changes even occur in prodromal PD $[4,14]$. There are also differences in the cognitive profiles of DLB and PD-D (Tab. 1). Smirnov et al. showed greater memory impairment in DLB and greater visuospatial impairment in PD-D [15]. Additionally, they showed that language function declined at a faster rate in DLB, while visuospatial and executive domains declined faster in PD-D [15].

\section{Multiple system atrophy (MSA)}

Multiple system atrophy (MSA) is another synucleinopathy that is histopathologically characterised by glial cytoplasmic inclusions (GGIs) rather than neuronal Lewy bodies. MSA shares some characteristics with PD and DLB, though the most recent diagnostic consensus statement currently lists dementia as a 'red flag' against a diagnosis of MSA, especially the cerebellar subtype (MSA-C) [16]. Our understanding of MSA-related cognitive change has expanded, and dementia has been identified in up to $31 \%$ of cases [17]. A large study of pathologically confirmed MSA showed mild, moderate, and severe cognitive impairment was present in $22 \%, 2 \%$, and $0.5 \%$ of cases, respectively [18]. The prevalence of cognitive impairment increases with disease duration and severity of motor impairment, with $50 \%$ of patients experiencing cognitive impairment after eight years of disease [19]. Patients with cognitive impairment have a greater burden of GCIs in limbic regions [20]. Several studies have demonstrated that the cognitive profile of MSA is predominantly characterised by a dysexecutive syndrome [17]. A cross-sectional study comparing the cognitive profiles of patients with MSA parkinsonian subtypes (MSA-P) and MSA-C found that both groups had executive function and visuospatial impairments, but attentional deficits were only present in the MSA-C cohort (Tab. 1) [19]. Kawai et al. compared the neuropsychological test scores and SPECT imaging of patients with MSA-P to MSA-C [21]. Patients with MSA-P had severe impairment of visuospatial and constructional function, verbal fluency, and executive function, while those with MSA-C had only involvement of visuospatial and constructional function, and to a milder degree than the MSA-P group. Pseudobulbar affect (PBA) is a more common feature of MSA-C than of MSA-P, and is attributed to disruption of cortico-ponto-cerebellar circuitry $[22,23]$.

\section{Progressive supranuclear palsy (PSP)}

Progressive supranuclear palsy (PSP) is an atypical parkinsonian disorder caused by $4 \mathrm{R}$ tau deposition within the brain. Individuals with PSP often experience more pronounced cognitive decline early in the disease course, which can help differentiate this disease from other parkinsonian disorders. Like the aforementioned parkinsonian syndromes, the cognitive profile of PSP is predominantly one of executive dysfunction
[24-26]. However, differential patterns of hypometabolism on ${ }^{18} \mathrm{~F}$-fluorodeoxyglucose positron emission tomography (FDG-PET) brain scans show that there is greater involvement of the frontal cortex than in MSA, DLB, or PD [27, 28]. This may account for some differences in executive domain testing compared to these other parkinsonian syndromes. Santangelo et al. showed that PSP patients scored lower on the Montreal Cognitive Assessment (MoCA) than patients with PD or MSA, though the latter was not significant, and the PSP cohort was older and less educated [29]. Those with PSP had more severe executive dysfunction characterised by deficits in spatial planning and set shifting (Tab. 1). This finding is consistent with other FDG-PET studies supporting the notion that executive dysfunction of PSP is related to frontal lobe dysfunction rather than pure subcortical dysfunction [30, 31].

Classic PSP, also known as Richardson's Syndrome (PSP-RS), is characterised by the presence of a vertical gaze supranuclear palsy and postural instability [32], although an accurate diagnosis of PSP is often delayed by several years because oculomotor abnormalities may be absent early in the disease [33]. There are several subtypes, including PSP with predominant parkinsonism (PSP-P), which can be especially difficult to differentiate from PD [34]. Neuropsychological assessments may be able to differentiate among PSP-RS, PSP-P, and PD as early phonological verbal fluency deficits are found to be more characteristic of PSP-RS while PSP-P patients may be more apathetic (Tab. 1) [35]. PBA is also common in PSP, probably due to pontine involvement $[22,23]$. A corticobasal syndrome (CBS) of parkinsonism with higher cortical features may also constitute a PSP subtype (PSP-CBS), wherein the cognitive profile is characterised by frontal executive dysfunction in addition to more posterior-based abnormalities, e.g. disorders of praxis, Gerstmann syndrome, etc. PSP with predominant speech and/or language dysfunction (PSP-SL) may have profound language dysfunction manifesting as apraxia of speech, dysprosody, or non-fluent aphasia [32, 34]. Some patients with PSP will have prominent frontal dysfunction in the form of reduced empathy, socially inappropriate behaviours, and impulse control (PSP-FTD) [34]. According to current diagnostic criteria, these cognitive predominant entities (PSP-CBS, PSP-SL, PSP-FTD) are condensed into PSP-F once there are sufficient accompanying features (oculomotor dysfunction, postural instability, or akinesia) to reach a diagnostic certainty of 'probable' [32]. PSP subtypes are often phenotypically similar to other neurodegenerative diseases.

\section{Corticobasal degeneration (CBD)}

Corticobasal degeneration (CBD) is a $4 \mathrm{R}$ tauopathy that can have substantial phenotypic overlap with PSP, but with distinct neuropathological differences [36]. CBD is regarded as a rare disease and accounts for only $4-6 \%$ of parkinsonism [37]. CBD specifically indicates a pathological diagnosis while the more accurate antemortem term is CBS, which can be caused by other pathologies, e.g. Alzheimer's Disease (AD), 
frontotemporal lobar degeneration with TDP-43, and PSP [38]. This results in an overdiagnosis of CBD, while phenotypic overlap with other neurodegenerative diseases may result in a missed diagnosis. The Mayo Clinic Florida brain bank found that only $37 \%$ of patients with pathologically confirmed CBD were clinically diagnosed with a CBS [39]. The most common misdiagnoses were PSP followed by $\mathrm{AD}$ and frontotemporal dementia. For these reasons, one should cautiously interpret CBD studies that lack pathological confirmation of disease.

As the name suggests, the symptoms of CBD localise to the cerebral cortex and basal ganglia. Hallmark features of the disease include asymmetric parkinsonism with higher cortical features, e.g. limb apraxia and cortical sensory loss (Tab. 1) [40]. Cognitive impairment is present in more than half of patients at the time of presentation and in $70 \%$ by the time of death [40]. Patients with CBD may have language and/or speech dysfunction, most often manifesting as a primary progressive aphasia or apraxia of speech, respectively [41]. Murray et al. also identified visuospatial difficulties in $40 \%$ of pathologically confirmed CBD cases at the time of symptom onset [42]. This was accompanied by acalculia, cortical sensory loss, memory impairment, and left/right discrimination. These patients underwent a second neuropsychological assessment roughly 20 months later, at which time these symptoms were more prevalent and $14.3 \%$ of patients had also developed lateralised neglect. A recent study found patients with CBS of known pathology to have significant asymmetric FDG-PET hypometabolism in frontoparietal regions, including the perirolandic area, basal ganglia, and thalamus [43]. Of the pathologies (CBS-CBD, CBS-PSP, and CBS-AD), CBS-CBD was associated with the greatest degree of hypometabolism in the basal ganglia bilaterally. Current diagnostic criteria account for atypical CBD cognitive profiles with frontal behavioural-spatial syndrome and nonfluent/agrammatic variant designations [40].

\section{Normal pressure hydrocephalus (NPH)}

Normal pressure hydrocephalus (NPH) is non-degenerative cause of parkinsonism. NPH is classically characterised by a clinical triad of gait abnormalities followed by cognitive impairment and urinary dysfunction. A Norwegian population-based study of 220,000 people found the prevalence and incidence of NPH to be 21.9 per 100,000 and 5.5 per 100,000 , respectively [44]. The cognitive impairment of NPH is commonly described as 'frontal-subcortical' based upon the presence of executive dysfunction, decreased attention and concentration, psychomotor slowing, and apathy [45]. However, this pattern of cognitive impairment is not specific for NPH. The degree of hydrocephalus and the presence of concurrent pathology influence this cognitive profile. It may be helpful to think of NPH as an 'inside out' disorder with cognitive impairment that is primarily localised to the subcortex. This is demonstrated with FDG-PET studies showing hypometabolism of the caudate and putamen while preserving the cortex [46]. Thus, cortical findings, such as anomia or apraxia, should steer the diagnosis towards alternative or co-existent pathologies of AD or CBD, respectively [47, 48].

\section{Other causes of parkinsonsim}

A working knowledge of non-degenerative causes of parkinsonism helps clinicians to identify treatable disorders. The designation of so-called 'vascular parkinsonism' has come under scrutiny due to the absence of specific abnormal structural imaging and poor correlation between brain MRI hyperintensities and clincopathological data [49]. Despite a lack of supporting pathology, cumulative leukoaraiosis has been implicated in causing symmetric predominantly lower body parkinsonism [50]. Similarly, subsequent subcortical cognitive impairment (Binswanger's Disease) is typically characterised by executive dysfunction more than memory impairment as well as slowed processing speed (Tab. 1) [50]. True vascular parkinsonism is caused by strategic infarctions involving the substantia nigra and/or nigrostriatal pathway, but sparing the striatum [49]. True vascular parkinsonism may be asymmetric and typically responds favourably to levodopa. Specific cognitive symptoms depend upon the infarct location, which can include brain regions outside of the basal ganglia.

Drug-induced parkinsonism can be difficult to distinguish from idiopathic PD, although clues such as a temporal relationship to starting dopamine blocking medications, poor levodopa response, and symmetric parkinsonism should increase the index of suspicion. DaTScan typically shows normal radioisotope uptake, consistent with preserved presynaptic dopaminergic function, in drug-induced parkinsonism and can be helpful in ruling out concurrent neurodegenerative parkinsonism, especially if symptoms are asymmetric [51-53]. Cognitively, patients may have comorbid psychiatric disease (those on antipsychotics) or gastrointestinal dysfunction (those on dopamine blocking antiemetics) and demonstrate a variety of medication-related side effects beyond parkinsonism, e.g. slowed processing and drowsiness.

\section{Parkinsonian gait disorders}

The human gait is a complex process that becomes remarkably efficient during the first years of life. Behind this relatively automatic process lie a huge number of complex neural processes that synchronise the activation and inhibition of specific muscle groups to allow for proper human locomotion. Parkinsonism can manifest with a variety of gait disturbances, and the ability to recognise and interpret these disturbances is a valuable tool for clinicians (Fig. 1).

\section{Gait manifestations of parkinsonism}

The prototypical parkinsonian stance and gait has been well appreciated since Sir William Richard Gowers's classic 


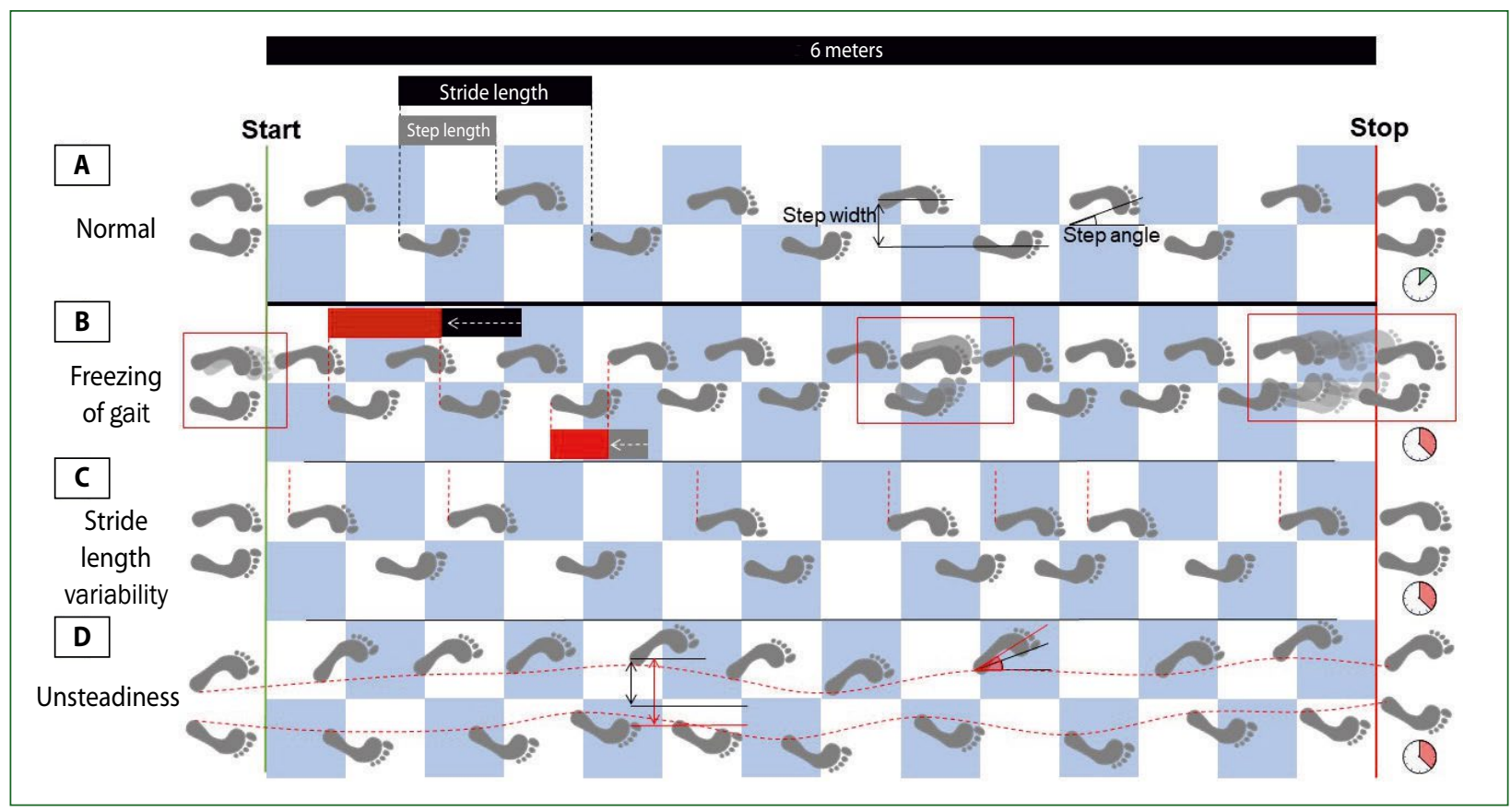

Figure 1. Common gait patterns seen with parkinsonian syndromes. Hypothetical gait profile that would be normal for 65-year-old woman (A). Illustrative examples of abnormal gait disturbances showing shortened step and stride lengths with multiple episodes of gait freezing occurring at gait initiation, mid-walk, and at time of desired gait termination with slow velocity (B), stride length variability and slowed velocity (C) and unsteadiness with widened base, increased step angle, lateral sway, and slowed velocity (D). Clock illustrations indicate that gait patterns of panels B-D are slow

1886 illustration of an individual with moderately severe PD in the pre-levodopa era [54]. From only two vantage points, one can appreciate an expressionless man with a camptocormic posture, short step length, and restricted arm movements. This phenotype is quickly recognised by novice clinicians; however, patients typically present well before the motor manifestations are so robust. More often, one must detect and interpret subtle deviations from the normal gait cycle in the context of multiple medical comorbidities to reach a diagnosis.

Parkinsonian gait disorders universally cause reduced gait velocity, the severity of which can be determined by comparison to age-based normative values [55]. Gait velocity is easy to measure and has significant correlations to functional independence, risk of falls, and QoL [56]. One should remember that gait cadence (steps/min) is separate from gait velocity, which measures distance, and that these two metrics do not necessarily correlate. Cadence is increased in most parkinsonian disorders, although it may be increased or decreased with NPH $[57,58]$.

Amplitude down-scaling is a hallmark feature of parkinsonian movements. Gait manifestations may include reduced step length and height during the swing period as well as reduced arm swing. NPH is an exceptional parkinsonian disorder and is sometimes referred to as lower body parkinsonism because arm swing is typically preserved [59]. Arm swing is most often symmetrically reduced in parkinsonian syndromes; however, asymmetry is expected in PD and CBS
$[40,60]$. In PD, the side with less swing generally corresponds to the side of greater bradykinesias and/or rigidity during isolated testing of movement and tone. Direct assessment is critically important because orthopaedic issues commonly cause asymmetrically reduced arm swing. The asymmetric arm swing of CBS may be more complicated as this can be confounded by the presence of dystonia, apraxia, or even alien limb syndrome [40]. In rare instances, this manifests as the more severely affected arm swinging with greater amplitude [61]. While PSP-RS tends to be a symmetric disorder, patients with PSP-P may have asymmetric arm swing [62]. Vascular parkinsonism resulting from an acute injury to the substantia nigra and/or nigrostriatal pathways will typically manifest with levodopa-responsive parkinsonism [63]. The so-called vascular parkinsonism due to cumulative leukoaraiosis is typically symmetrical, preferentially involves the legs, and does not respond to levodopa [49].

\section{Balance abnormalities of parkinsonism}

Imbalance can be a substantial contributor to the gait abnormalities of parkinsonism, and one should consider the numerous common contributors to imbalance, e.g. peripheral neuropathy, medications, peripheral vestibular disorders, etc. These alternative causes of imbalance can typically be detected with focused neurological assessment. A wide step width is a nonspecific marker of instability and is more common in atypical parkinsonian syndromes compared to $\mathrm{PD}$, in which 


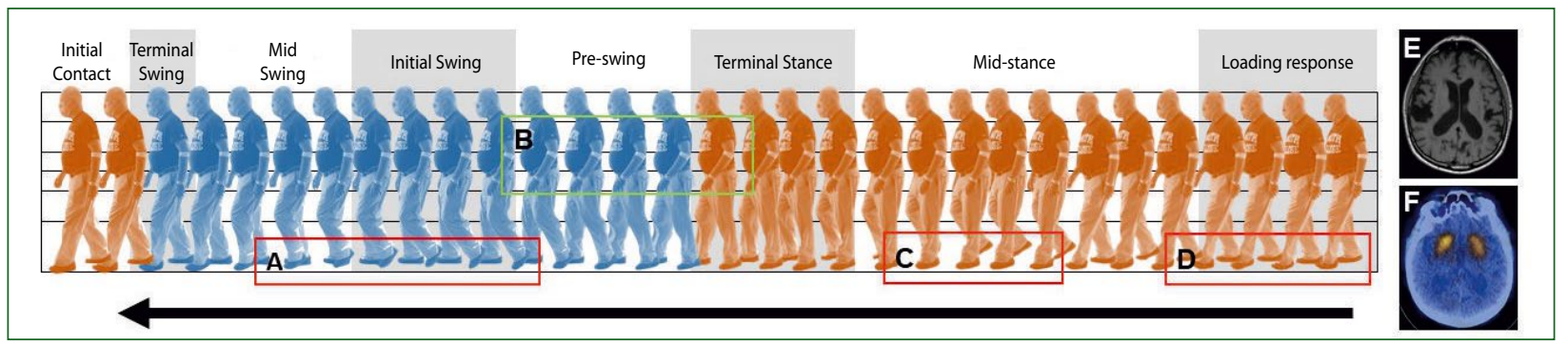

Figure 2. Gait cycle in normal pressure hydrocephalus. Pre-shunt gait cycle of 79-year-old man with shunt-responsive normal pressure hydrocephalus illustrating decreased step height (A), preserved arm swing (B), outward foot rotation (C), and short stride length (D). Axial T1-weighted MRI of patient's brain demonstrates ventriculomegaly and sulcal trapping (E) and his DaTScan shows preserved radiotracer in basal ganglia bilaterally (F). Images were acquired at 29 frames per second

Table 2. Gait features of parkinsonian disorders

\begin{tabular}{|c|c|c|c|c|c|c|c|c|c|c|c|c|}
\hline & \multirow[t]{2}{*}{ PD } & \multirow[t]{2}{*}{ DLB } & \multicolumn{2}{|c|}{ MSA } & \multicolumn{4}{|c|}{ PSP } & \multirow[t]{2}{*}{ CBD } & \multirow[t]{2}{*}{ NPH } & \multirow{2}{*}{$\begin{array}{l}\text { Vasc- } \\
\text {-Park }\end{array}$} & \multirow{2}{*}{$\begin{array}{l}\text { Med- } \\
\text {-Park }\end{array}$} \\
\hline & & & $\mathbf{P}$ & $C$ & RS & $\mathbf{P}$ & $C$ & CBS & & & & \\
\hline Gait velocity & $\downarrow$ & $\downarrow$ & $\downarrow$ & $\downarrow$ & $\downarrow$ & $\downarrow$ & $\downarrow$ & $\downarrow$ & $\downarrow$ & $\downarrow$ & $\downarrow$ & $\downarrow$ \\
\hline Stride length & $\downarrow$ & $\downarrow$ & $\downarrow$ & $\downarrow$ & $\downarrow$ & $\downarrow$ & $\downarrow$ & $\downarrow$ & $\downarrow$ & $\downarrow$ & $\downarrow$ & $\downarrow$ \\
\hline Asymmetry & + & - & - & - & - & + & - & + & + & - & \pm & \pm \\
\hline Arm swing & $\downarrow$ & $\downarrow$ & $\downarrow$ & $\downarrow$ & $\downarrow$ & $\downarrow$ & $\downarrow$ & $\uparrow \downarrow$ & $\uparrow \downarrow$ & $\mathrm{Nml} / \downarrow$ & $\mathrm{Nml} / \downarrow$ & $\downarrow$ \\
\hline FoG & $t^{*}$ & + & + & \pm & + & + & + & + & + & + & + & + \\
\hline Ataxia & - & - & - & + & - & - & + & - & - & - & \pm & - \\
\hline Cadence & $\uparrow$ & $\uparrow$ & $\uparrow$ & $\uparrow$ & $\uparrow$ & $\uparrow$ & $\uparrow$ & $\uparrow$ & $\uparrow$ & $\uparrow \downarrow$ & $\uparrow$ & $\uparrow \downarrow$ \\
\hline Step width & $\mathrm{Nml} / \downarrow$ & $\mathrm{Nml} / \downarrow$ & $\mathrm{Nml} / \downarrow$ & $\uparrow$ & $\uparrow$ & $\mathrm{Nml} / \downarrow$ & $\uparrow$ & $\mathrm{Nml} / \downarrow$ & $\mathrm{Nml} / \downarrow$ & $\uparrow$ & $\mathrm{Nml} / \downarrow$ & $\mathrm{Nml} / \downarrow$ \\
\hline Levodopa response & + & + & \pm & - & \pm & \pm & - & - & - & - & + & \pm \\
\hline
\end{tabular}

$\downarrow$ decreased; $\uparrow$ increased; $\uparrow \downarrow$ variably increased or decreased; + present; - absent; Nml normal; ${ }^{\natural}$ Late in disease

CBD - corticobasal degeneration; DLB - dementia with Lewy bodies; Med-Park - medication-induced parkinsonism; MSA — multiple system atrophy; MSA-C — MSA cerebellar subtype; MSA-P — MSA parkinsonian subtype; $\mathrm{NPH}$ — normal pressure hydrocephalus; PD — Parkinson's Disease; PSP — progressive supranuclear palsy; PSP-CBS — PSP corticobasal syndrome subtype; PSP-P — PSP with predominant parkinsonism; PSP-RS — PSP Richardson's Syndrome; Vasc-Park — vascular parkinsonism due to basal ganglia infarct

the base is typically normal (Fig. 1D) [64, 65]. Abdo et al. exploited this difference by showing greater difficulty with tandem walking for those with atypical parkinsonian syndromes compared to PD [66]. Patients with NPH may have a wide step width and increased foot angle (degree of external rotation), both of which are considered compensatory balance-related gait parameters [67] (Fig. 2).

The impairment of postural reflexes is a major cause of falls. Postural instability is usually a finding of advanced PD; however, it may occur early in the disease course of other parkinsonian syndromes including PSP, NPH, and a subset of PD characterised by postural instability and gait disorder [34, $58,68-70]$. Early falls may be an indicator of impaired postural reflexes and can be directly assessed with the shoulder pull test, which is a component of several clinical rating scales, e.g. the Movement Disorders Society Unified Parkinson's Disease Rating Scale (MDS-UPDRS). The manoeuvre involves applying a brief, posteriorly directed, pulling force to a patient's shoulders. The patient is instructed to maintain their balance. Due to variability in the examiner's pulling force, this test may be subject to interrater variability [71]. The integrity of postural reflexes may also be tested by the 'push and release test', which requires the patient to overcome a sudden loss of support when pushing posteriorly with the upper back against resistance. Gait variability is related to postural reflexes and fall risk [72]. An oscillatory type of gait variability known as the 'sequence effect' manifests in a progressive reduction in amplitude eventually leading to akinesia [73]. This may be the gait correlate of finger tap dampening.

\section{Freezing of gait (FoG)}

Freezing of gait (FoG) is a common episodic phenomenon in parkinsonian disorders characterised by the sudden arrest of the gait cycle's forward progression (Fig. 1B). FoG may occur in all forms of parkinsonism, and the disease stage of FoG onset can help to differentiate between specific diagnoses. In PD, FoG may affect $20-25 \%$ of individuals with early-stage disease, and up to $80 \%$ of those in the late stages [74-76]. FoG is more common and occurs earlier in PSP, MSA, vascular parkinsonism, higher level gait disorders, DLB, CBD, and NPH [58, 77-79]. Pure akinesia with gait freezing (PAGF), also known as primary progressive FoG, is a clinical syndrome of isolated difficulties involving gait initiation and freezing while walking [80]. PAGF is a PSP subtype (PSP-PAGF) and 
highly predictive of PSP-tau pathology, although other causes, including pallidonigroluysian degeneration and DLB, have been identified [34, 80, 81]. Some patients with PAGF will develop additional clinical symptoms leading to a clinical diagnosis of more typical PSP or CBD [81].

Our understanding of the mechanisms underlying FoG is incomplete, and there are probably several contributors to FoG based upon the variety of precipitating circumstances and variability in responses to treatments. FoG often occurs during gait initiation, turns, navigating tight spaces, and dual tasking [82]. PD-associated FoG may or may not be levodopa-responsive and among those who show a response, FoG may respond favourably or paradoxically worsen in the "ON" state $[83,84]$.

This suggests that FoG is more than a severe form of bradykinesia, which is levodopa responsive in PD, and may receive contributions from dysregulated neurotransmitters other than dopamine [84].

Patients with FoG also have increased step time variability, abnormal bilateral coordination during turns, and a greater cadence [82, 84-86]. Interestingly, cadence-reducing external cues diminish FoG during turns [87]. This has contributed to the 'threshold model' of FoG which states that accumulation of motor deficits eventually reaches a threshold at which motor arrest occurs [88]. The 'interference model' suggests that there is a finite amount of cerebral resources that, when exhausted, result in breakdown of proper locomotion [89]. This model accounts for nonuniform involvement of parallel cortico-striato-thalamocortical loops (oculomotor, sensorimotor, associate, and limbic) and is illustrated by increased freezing with dual tasking or navigating obstacles [11,90]. The 'cognitive model' considers the lost automaticity of cerebral calculations required for normal gait [91]. The loss of automaticity requires a cognitive compensation dependent on adequate executive function. The result is an inverse relationship between executive function and the likelihood of freezing. This is evidenced by studies showing that persons with PD-associated FoG have more pronounced executive dysfunction than non-freezers [92]. Others have proposed a 'decoupling model', which proposes a disconnect between motor planning and movement execution [93]. This can be exemplified by difficulties with gait ignition. Patients may describe their feet as 'glued to the floor,' This is associated with abnormal anticipatory postural adjustments (APAs), which may accompany other FoG phenomenology, e.g. knee trembling, improperly timed stepping, or step arrest. Nieuwboer et al. studied the electromyographic profile of FoG, and found premature onset and termination of activity in the tibialis anterior and gastrocnemius muscles prior to freezing [94].

\section{Multifactorial gait dysfunction}

In rare exceptions, a patient will have a gait disorder purely due to a single factor. As such, clinicians must cautiously interpret all findings and resist the urge to attribute all gait abnormalities to a single pathology. For instance, orthopaedic shoulder pathology is a very common cause of reduced arm swing and can be misinterpreted as the harbinger of an impending neurodegenerative disease. Additional pathologies, e.g. longstanding essential tremor manifesting with a rest component, complicates the clinical scenario and increases the importance of sound clinical judgement. Most parkinsonian patients are elderly, so the possibility for multiple pathologies is substantial. Nevertheless, the expansive reach of some neurodegenerative conditions can lead to a variety of gait disturbances that are explained by a solitary pathological substrate.

The gait disturbances of PSP warrant special attention because there are several contributory factors leading to imbalance and resulting in early falls. Rigidity is a cardinal feature of parkinsonism, but the distribution in PSP is especially pertinent because it involves the trunk and neck more than other parkinsonian syndromes. While the trunk and neck are not required for gait, changes to the so-called 'passenger unit' (head, arms, trunk) can cause deviations from one's normal gait profile. Perhaps the most common example is obesity, which causes decreased ankle plantar flexion at toe-off, reduced hip flexion at heel strike and mid swing, and reduced hip extension during push off, resulting in altered anteroposterior and mediolateral ground reaction forces [95]. The axial and nuchal rigidity of PSP limits one's ability to rapidly compensate for abrupt directional changes that occur with various balance challenges, of which there are many in PSP. Patients often tend towards retropulsion (falling backwards), which may be partially compensated by a flexed trunk or camptocormia. However, this anterior tilt can compound the risk of falls due to FoG, which is very common in PSP [77]. The combination of these features may manifest as a cautious and unsteady gait sometimes described as a 'drunken sailor' [96]. PSP gait impairment is further complicated by frontally based cognitive impairment, which may impact upon a patient's judgement and subsequent underestimation of their ambulatory difficulties. The 'rocket sign' is a manifestation of impulsivity that has been described in PSP, wherein a patient falls back into their chair after rapidly and incautiously arising from a seated position [96].

\section{The gait-cognition relationship}

In recent years, our understanding of the complex relationship between gait and cognitive function has increased, largely due to studies specifically targeting this question using improved measurement techniques, e.g. quantitative gait analysis, and advanced neuroimaging. Dual task (DT) paradigms challenge the subject with a cognitively demanding task, e.g. serial sevens, while performing a gait task. Most reports demonstrate that DT paradigms negatively impact upon gait variables of healthy adults, more so in older individuals [97]. Studies of individuals with mild cognitive impairment, Alzheimer's Disease, and PD have shown that the degree of gait worsening with DT is directly related to the degree of cognitive impairment [98]. 
Executive dysfunction is the most widely appreciated correlate of gait impairment, although inverse associations with attention, visuospatial awareness and memory have also been demonstrated [98]. These effects also expand beyond the dopaminergic system, with increasing evidence pointing towards cholinergic dysfunction, particularly the cholinergic basal forebrain and pedunculopontine nucleus (PPN) [98, 99]. A recent study of $P D$ participants and controls found that smaller nucleus basalis of Meynert (NBM) volumes, measured utilising 3-tesla MRI, predicted increased step time variability and shortened swing time, while a decreased posterior portion NBM volume predicted shortened step length and increased step time variability [99]. A deeper understanding of the cholinergic role would have direct implications for treatments, and acetylcholinesterase inhibitors have been shown to improve some PD mobility measures [98]. Moreover, the PPN has been implicated in PD-related FoG and continues to be investigated as a target for deep brain stimulation [100].

\section{Measuring cognition and gait}

Cognitive and gait impairments of parkinsonian disorders may be subtle. This underlines the importance of applying reliable measurement tools and recognising their limitations.

\section{Considerations of cognitive measures}

The most detailed measurement of cognitive function comes through highly detailed neuropsychometric testing administered by a trained psychometrist and interpreted by a neuropsychologist. The details of designing a neuropsychological assessment are beyond the scope of this article; however, one should appreciate that neuropsychologists tailor their testing battery to the clinical context after interviewing the patient to determine which cognitive domains are likely to be affected. This increases the likelihood of achieving results with localising value and identifying cognitive profiles that support or refute items in the differential diagnosis.

The author recognises that detailed neuropsychometric testing may be impractical and indeed unnecessary in many instances. Therefore, it is important to consider the specific clinical context when choosing the most appropriate cognitive screening test. Rather than a one size fits all approach, clinicians should choose their bedside cognitive screen in the same manner that a neuropsychologist would choose their patient-specific testing battery. In the case of parkinsonian disorders, one would expect dysfunction of cognitive processes that are dependent upon frontal and subcortical structures. The Folstein Mini-Mental Status Examination (MMSE) is not especially sensitive to executive function and is therefore insensitive at detecting mild cognitive impairment (MCI) in PD [101]. The Montreal Cognitive Assessment (MoCA) and the Kokmen Short Test of Mental Status (STMS) are better assessments in this case. The Movement Disorders Society considers the MoCA, PD Cognitive Rating Scale (PD-CRS), Scales for Outcomes of Parkinson's Disease-Cognition (SCOPA-COG), and Mattis Dementia Rating Scale (MDRS) to be appropriate cognitive screens for the assessment of PD [9].

Cognitive screens also help to identify elements of a cognitive profile that are inconsistent with a specific diagnosis. For instance, identification of anomia in a person suspected of having NPH should raise suspicion for $\mathrm{AD}[47,48]$. This finding should not immediately result in a diagnosis swap, especially if there is sufficient supporting evidence to diagnose $\mathrm{NPH}$. Rather, the clinician should consider the possibility of concurrent $\mathrm{AD}$ pathology, which occurs in roughly one in three NPH cases [102]. The prospect of dual pathology extends beyond this example, and should be considered when phenotypes deviate significantly from classical norms.

When approaching a patient with parkinsonism, clinicians should be especially sensitive to detect cognitive impairment even in the absence of overt concerns by the patient or informant. A subtle abnormality of executive function, e.g. being slower to complete multi-step tasks or a deviation from one's previous level of conversational engagement, may be an important clue to unlock the correct diagnosis and inform the clinician of an increased fall risk [98].

Given the challenge of accurately identifying executive dysfunction from interview alone, the author recommends administering a bedside cognitive screen that is sensitive to executive dysfunction to all patients presenting with parkinsonism. It is often useful to measure the time taken to complete such a cognitive screen. Most bedside screens should be completed within 10 minutes. Even a high score with a slow completion time should alert the clinician to a potential problem, whether cognitive, psychiatric, or purely motor. To minimise motor confounders, cognitive testing should occur when patients are in a levodopa "ON" state if applicable. If the cognitive profile remains unclear after a cognitive screen, then a formal neuropsychological evaluation is prudent. This serves a threefold purpose, by providing a cognitive baseline, screening for pertinent comorbid psychiatric factors, and localising the cognitive profile.

\section{Considerations of gait measures}

Gait assessments range from casually observing a patient en route to the examination room to formal gait assessment in a motion analysis laboratory. The approach to the assessment of gait parallels that of cognitive assessments. Clinicians will always obtain a thorough history and examination, but a more formal gait analysis may be required if diagnostic ambiguity persists. There are many useful scales for characterising and determining the severity of gait or balance problems. These are most useful for finding associations with QoL or functional status. The timed-up-and-go (TUG) consists of standing up from a chair, walking forward 3 metres, turning, returning to the chair, and sitting down. A TUG time of $\geq 12.6$ seconds has been associated with future falls [103]. While useful, abnormalities on assessments like the TUG and MDS-UPDRS 
or questionnaires like the Freezing of Gait Questionnaire (FOGQ) are nonspecific.

Formal gait assessments may incorporate a variety of technologies, e.g. electromyography, accelerometry, gyroscopy, pressure sensing, and infrared three-dimensional video. A detailed description of motion analysis is beyond the scope of this review, but these modalities provide objective information about various aspects of one's gait, e.g. muscle activity, kinematics, and kinetics. Biomechanical information of such granularity can inform treatment decisions such as orthopaedic surgical planning for patients with cerebral palsy [104].

Detailed gait analysis is now being more regularly applied to neurodegenerative diseases. McArdle et al. utilised an instrumented walkway to identify greater gait asymmetry and variability in patients with LBD compared to AD [105]. As such, there is potential for identifying pathological signatures of gait that can facilitate the diagnostic process. Formal gait analysis may also be useful for sorting out the various contributory elements of a multifactorial gait disorder.

The use of wearable sensors has gained great popularity due to the greater convenience and lower costs relative to expensive laboratory-based motion analysis. A recent study found that application of a single wearable accelerometer could differentiate between AD, DLB, and PD [106]. While the potential for broadly applying these technologies is exciting in the field of parkinsonian disorders, studies should be cautiously interpreted because many technologies are not independently verified or validated [107]. Nevertheless, the global shift towards telemedicine in the wake of COVID-19 has left a field ripe with possibilities for remote assessment of movement disorders.

\section{Conclusion}

Our understanding of parkinsonian conditions has increased substantially in recent years. Patients with parkinsonism invariably have disturbances, at times only subtle, of cognition and/or gait. Understanding the various manifestations of these disturbances can guide clinicians toward a more accurate and timely diagnosis and inform treatment decisions.

This review provides a summary of cognitive and gait manifestations of the most common causes of parkinsonism and discusses important considerations when assessing these parkinsonian attributes. There is much to learn about parkinsonism and the mechanisms underlying its phenotypic manifestations. By leveraging emerging technologies to carefully evaluate these phenotypes, we will advance the field toward a deeper understanding of parkinsonian disorders and improve patient care.

Acknowledgements: Special thank you to Wade Tipton and Megan Tipton for assistance with figure artwork.

Conflict of interest: None.

\section{References}

1. Witjas T, Kaphan E, Azulay JP, et al. Nonmotor fluctuations in Parkinson's disease: frequent and disabling. Neurology. 2002; 59(3): 408-413, doi: 10.1212/wnl.59.3.408, indexed in Pubmed: 12177375.

2. Corallo F, De Cola MC, Lo Buono V, et al. Observational study of quality of life of Parkinson's patients and their caregivers. Psychogeriatrics. 2017; 17(2): 97-102, doi: 10.1111/psyg.12196, indexed in Pubmed: 27338524.

3. Aarsland D, Muniz G, Matthews F. Nonlinear decline of mini-mental state examination in Parkinson's disease. Mov Disord. 2011; 26(2): 334-337, doi: 10.1002/mds.23416, indexed in Pubmed: 20960482.

4. Aarsland D, Brønnick K, Larsen JP, et al. Norwegian ParkWest Study Group. Cognitive impairment in incident, untreated Parkinson disease: the Norwegian ParkWest study. Neurology. 2009; 72(13): 1121-1126, doi: 10.1212/01.wnl.0000338632.00552.cb, indexed in Pubmed: 19020293.

5. Pedersen KF, Larsen JP, Tysnes OB, et al. Natural course of mild cognitive impairment in Parkinson disease: a 5-year population-based study. Neurology. 2017; 88(8): 767-774, doi: 10.1212/ WNL.0000000000003634, indexed in Pubmed: 28108638.

6. Hoogland J, Boel JA, de Bie RMA, et al. MDS Study Group "Validation of Mild Cognitive Impairment in Parkinson Disease". Risk of Parkinson's disease dementia related to level I MDS PD-MCI. Mov Disord. 2019; 34(3): 430-435, doi: 10.1002/mds.27617, indexed in Pubmed: 30653248.

7. Aarsland D, Andersen K, Larsen JP, et al. Risk of dementia in Parkinson's disease: a community-based, prospective study. Neurology. 2001; 56(6): 730-736, doi: 10.1212/wnl.56.6.730, indexed in Pubmed: 11274306.

8. Lang S, Hanganu A, Gan LS, et al. Network basis of the dysexecutive and posterior cortical cognitive profiles in Parkinson's disease. Mov Disord. 2019; 34(6): 893-902, doi: 10.1002/mds.27674, indexed in Pubmed: 30924964.

9. Litvan I, Goldman JG, Tröster Al, et al. Diagnostic criteria for mild cognitive impairment in Parkinson's disease: Movement Disorder Society Task Force guidelines. Mov Disord. 2012; 27(3): 349-356, doi: 10.1002/mds.24893, indexed in Pubmed: 22275317.

10. Williams-Gray $\mathrm{CH}$, Evans JR, Goris An, et al. The distinct cognitive syndromes of Parkinson's disease: 5 year follow-up of the CamPalGN cohort. Brain. 2009; 132(Pt 11): 2958-2969, doi: 10.1093/brain/ awp245, indexed in Pubmed: 19812213.

11. Kehagia AA, Barker RA, Robbins TW. Cognitive impairment in Parkinson's disease: the dual syndrome hypothesis. Neurodegener Dis. 2013; 11(2): 79-92, doi: 10.1159/000341998, indexed in Pubmed: 23038420.

12. Knox MG, Adler $\mathrm{CH}$, Shill $\mathrm{HA}$, et al. Neuropathological findings in Parkinson's disease with mild cognitive impairment. Mov Disord. 2020; 35(5): 845-850, doi: 10.1002/mds.27991, indexed in Pubmed: 32034933.

13. McKeith I, Boeve B, Dickson D, et al. Diagnosis and management of dementia with Lewy bodies. Neurology. 2017; 89(1): 88-100, doi: 10.1212/wnl.0000000000004058, indexed in Pubmed: 28592453.

14. Chahine LM, Weintraub D, Hawkins KA, et al. PARS Investigators. Cognition in individuals at risk for Parkinson's: Parkinson associated risk syndrome (PARS) study findings. Mov Disord. 2016; 31(1): 86-94, doi: 10.1002/mds.26373, indexed in Pubmed: 26293177.

15. Smirnov DS, Galasko D, Edland SD, et al. Cognitive decline profiles differ in Parkinson disease dementia and dementia with Lewy 
bodies. Neurology. 2020; 94(20): e2076-e2087, doi: 10.1212/ WNL.0000000000009434, indexed in Pubmed: 32332125.

16. Gilman S, Wenning GK, Low PA, et al. Second consensus statement on the diagnosis of multiple system atrophy. Neurology. 2008; 71(9): 670-676, doi: 10.1212/01.wnl.0000324625.00404.15, indexed in Pubmed: 18725592.

17. Stankovic I, Krismer F, Jesic A, et al. Movement Disorders Society MSA (MODIMSA) Study Group. Cognitive impairment in multiple system atrophy: a position statement by the Neuropsychology Task Force of the MDS Multiple System Atrophy (MODIMSA) study group. Mov Disord. 2014; 29(7): 857-867, doi: 10.1002/mds.25880, indexed in Pubmed: 24753321.

18. Wenning GK, Tison F, Ben Shlomo Y, et al. Multiple system atrophy: a review of 203 pathologically proven cases. Mov Disord. 1997; 12(2): 133147, doi: 10.1002/mds.870120203, indexed in Pubmed: 9087971.

19. Barcelos LB, Saad F, Giacominelli C, et al. Neuropsychological and clinical heterogeneity of cognitive impairment in patients with multiple system atrophy. Clin Neurol Neurosurg. 2018; 164: 121-126, doi: 10.1016/j.clineuro.2017.10.039, indexed in Pubmed: 29223069.

20. Koga S, Parks A, Dickson DW, et al. Profile of cognitive impairment and underlying pathology in multiple system atrophy. Mov Disord. 2017; 32(3): 405-413, doi: 10.1002/mds.26874, indexed in Pubmed: 27859650.

21. Kawai $Y$, Suenaga M, Takeda A, et al. Cognitive impairments in multiple system atrophy: MSA-C vs MSA-P. Neurology. 2008; 70(16, Part 2): 1390-1396, doi: 10.1212/01.wnl.0000310413.04462.6a.

22. Hakimi M, Maurer CW. Pseudobulbar affect in parkinsonian disorders: a review. J Mov Disord. 2019; 12(1): 14-21, doi: 10.14802/ jmd.18051, indexed in Pubmed: 30732430.

23. Floeter MK, Katipally R, Kim MP, et al. Impaired corticopontocerebellar tracts underlie pseudobulbar affect in motor neuron disorders. Neurology. 2014; 83(7): 620-627, doi: 10.1212/ WNL.0000000000000693, indexed in Pubmed: 25008395.

24. Lange KW, Tucha O, Alders GL, et al. Differentiation of parkinsonian syndromes according to differences in executive functions. J Neural Transm (Vienna). 2003; 110(9): 983-995, doi: 10.1007/s00702003-0011-0, indexed in Pubmed: 12938023.

25. Monza D, Soliveri P, Radice D, et al. Cognitive dysfunction and impaired organization of complex motility in degenerative parkinsonian syndromes. Arch Neurol. 1998; 55(3): 372-378, doi: 10.1001/archneur.55.3.372, indexed in Pubmed: 9520011.

26. Robbins TW, James M, Owen AM, et al. Cognitive deficits in progressive supranuclear palsy, Parkinson's disease, and multiple system atrophy in tests sensitive to frontal lobe dysfunction. J Neurol Neurosurg Psychiatry. 1994; 57(1): 79-88, doi: 10.1136/jnnp.57.1.79, indexed in Pubmed: 8301310.

27. Hellwig $S$, Amtage $F$, Kreft A, et al. $\left[{ }^{18} \mathrm{~F}\right] \mathrm{FDG}-\mathrm{PET}$ is superior to [123!] IBZM-SPECT for the differential diagnosis of parkinsonism. Neurology. 2012; 79(13): 1314-1322, doi: 10.1212/WNL.0b013e31826c1b0a, indexed in Pubmed: 22914831.

28. Zhao P, Zhang B, Gao S. 18[F]-FDG PET study on the Idiopathic Parkinson's disease from several parkinsonian-plus syndromes. Parkinsonism \& Related Disorders. 2012; 18: S60-S62, doi: 10.1016/ s1353-8020(11)70020-7.

29. Santangelo G, Cuoco S, Pellecchia MT, et al. Comparative cognitive and neuropsychiatric profiles between Parkinson's disease, multiple system atrophy and progressive supranuclear palsy. J Neurol. 2018; 265(11): 2602-2613, doi: 10.1007/s00415-018-9038-x, indexed in Pubmed: 30178175.
30. Boxer A, Yu JT, Golbe L, et al. Advances in progressive supranuclear palsy: new diagnostic criteria, biomarkers, and therapeutic approaches. Lancet Neurol. 2017; 16(7): 552-563, doi: 10.1016/s14744422(17)30157-6, indexed in Pubmed: 28653647.

31. Tang C, Poston $\mathrm{K}$, Eckert $\mathrm{T}$, et al. Differential diagnosis of parkinsonism: a metabolic imaging study using pattern analysis. Lancet Neurol. 2010; 9(2): 149-158, doi: 10.1016/s1474-4422(10)70002-8, indexed in Pubmed: 20061183.

32. Höglinger GU, Respondek G, Stamelou M, et al. Movement Disorder Society-endorsed PSP Study Group. Clinical diagnosis of progressive supranuclear palsy: the movement disorder society criteria. Mov Disord. 2017; 32(6): 853-864, doi: 10.1002/mds.26987, indexed in Pubmed: 28467028.

33. Phokaewvarangkul 0 , Bhidayasiri R. How to spot ocular abnormalities in progressive supranuclear palsy? A practical review. Transl Neurodegener. 2019; 8: 20, doi: 10.1186/s40035-019-0160-1, indexed in Pubmed: 31333840.

34. Respondek G, Höglinger GU. The phenotypic spectrum of progressive supranuclear palsy. Parkinsonism Relat Disord. 2016; 22(Suppl 1): S34-S36, doi: 10.1016/j.parkreldis.2015.09.041, indexed in Pubmed: 26421392.

35. Pellicano C, Assogna F, Cellupica N, et al. Neuropsychiatric and cognitive profile of early Richardson's syndrome, progressive supranuclear Palsy-parkinsonism and Parkinson's disease. Parkinsonism Relat Disord. 2017; 45: 50-56, doi: 10.1016/j.parkreldis.2017.10.002, indexed in Pubmed: 29037499.

36. Dickson DW. Neuropathologic differentiation of progressive supranuclear palsy and corticobasal degeneration. J Neurol. 1999; 246(Suppl 2): ॥6-II5, doi: 10.1007/BF03161076, indexed in Pubmed: 10525997.

37. Constantinides VC, Paraskevas GP, Paraskevas PG, et al. Corticobasal degeneration and corticobasal syndrome: a review. Clin Park Relat Disord. 2019; 1: 66-71, doi: 10.1016/j.prdoa.2019.08.005, indexed in Pubmed: 34316603.

38. Whitwell JL, Jack CR, Boeve BF, et al. Imaging correlates of pathology in corticobasal syndrome. Neurology. 2010; 75(21): 1879-1887, doi: 10.1212/WNL.0b013e3181feb2e8, indexed in Pubmed: 21098403.

39. Koga S, Kouri N, Walton RL, et al. Corticobasal degeneration with TDP-43 pathology presenting with progressive supranuclear palsy syndrome: a distinct clinicopathologic subtype. Acta Neuropathol. 2018; 136(3): 389-404, doi: 10.1007/s00401-018-1878-z, indexed in Pubmed: 29926172.

40. Armstrong MJ, Litvan I, Lang AE, et al. Criteria for the diagnosis of corticobasal degeneration. Neurology. 2013; 80(5): 496-503, doi: 10.1212/WNL.0b013e31827f0fd1, indexed in Pubmed: 23359374.

41. Josephs KA, Duffy JR, Strand EA, et al. Clinicopathological and imaging correlates of progressive aphasia and apraxia of speech. Brain. 2006; 129(Pt 6): 1385-1398, doi: 10.1093/brain/awl078, indexed in Pubmed: 16613895.

42. Murray R, Neumann M, Forman MS, et al. Cognitive and motor assessment in autopsy-proven corticobasal degeneration. Neurology. 2007; 68(16): 1274-1283, doi: 10.1212/01. wnl.0000259519.78480.c3, indexed in Pubmed: 17438218.

43. Pardini M, Huey ED, Spina S, et al. FDG-PET patterns associated with underlying pathology in corticobasal syndrome. Neurology. 2019; 92(10): e1121-e1135, doi: 10.1212/WNL.0000000000007038, indexed in Pubmed: 30700592.

44. Brean A, Eide PK. Prevalence of probable idiopathic normal pressure hydrocephalus in a Norwegian population. Acta Neurol Scand. 2008; 
118(1): 48-53, doi: 10.1111/j.1600-0404.2007.00982.x, indexed in Pubmed: 18205881.

45. Picascia M, Zangaglia R, Bernini S, et al. A review of cognitive impairment and differential diagnosis in idiopathic normal pressure hydrocephalus. Funct Neurol. 2015; 30(4): 217-228, doi: 10.11138/ fneur/2015.30.4.217, indexed in Pubmed: 26727700.

46. Townley RA, Botha H, Graff-Radford J, et al. F-FDG PET-CT pattern in idiopathic normal pressure hydrocephalus. Neuroimage Clin. 2018; 18: 897-902, doi: 10.1016/j.nicl.2018.02.031, indexed in Pubmed: 29876274.

47. Graff-Radford NR, Godersky JC, Jones MP. Variables predicting surgical outcome in symptomatic hydrocephalus in the elderly. Neurology. 1989; 39(12): 1601-1604, doi: 10.1212/wnl.39.12.1601, indexed in Pubmed: 2586777.

48. De Mol J. [Prognostic factors for therapeutic outcome in normal-pressure hydrocephalus. Review of the literature and personal study]. Acta Neurol Belg. 1985; 85(1): 13-29, indexed in Pubmed: 3984674.

49. Vizcarra JA, Lang $A E$, Sethi $K D$, et al. Vascular parkinsonism: deconstructing a syndrome. Mov Disord. 2015; 30(7): 886-894, doi: 10.1002/mds.26263, indexed in Pubmed: 25997420.

50. Mendez MF, Cherrier MM, Perryman KM. Differences between Alzheimer's disease and vascular dementia on information processing measures. Brain Cogn. 1997; 34(2): 301-310, doi: 10.1006/ brcg.1997.0923, indexed in Pubmed: 9220093.

51. Gajos A, Dąbrowski J, Bieńkiewicz M, et al. The symptoms asymmetry of drug-induced parkinsonism is not related to nigrostriatal cell degeneration: a SPECT-DaTSCAN study. Neurol Neurochir Pol. 2019; 53(4): 311-314, doi: 10.5603/PJNNS.a2019.0031, indexed in Pubmed: 31441498.

52. Gajos A, Dąbrowski J, Bieńkiewicz M, et al. Should non-movement specialists refer patients for SPECT-DaTSCAN? Neurol Neurochir Pol. 2019; 53(2): 138-143, doi: 10.5603/PJNNS.a2019.0011, indexed in Pubmed: 30855703.

53. Barc K, Kuźma-Kozakiewicz M. Positron emission tomography neuroimaging in neurodegenerative diseases: Alzheimer's disease, Parkinson's disease, and amyotrophic lateral sclerosis. Neurol Neurochir Pol. 2019; 53(2): 99-112, doi: 10.5603/PJNNS.a2019.0013, indexed in Pubmed: 30855701.

54. Gowers WR. A manual of diseases of the nervous system. https:// www.worldcat.org/title/manual-of-diseases-of-the-nervous-system/ oclc/14805157 (August 22, 2021).

55. Lusardi M, Pellecchia G, Schulman M. Functional performance in community living older adults. Journal of Geriatric Physical Therapy. 2003; 26(3): 14-22, doi: 10.1519/00139143-200312000-00003.

56. Middleton A, Fritz SL, Lusardi M. Walking speed: the functional vital sign. J Aging Phys Act. 2015; 23(2): 314-322, doi: 10.1123/ japa.2013-0236, indexed in Pubmed: 24812254.

57. Lim YH, Ko PW, Park KS, et al. Quantitative gait analysis and cerebrospinal fluid tap test for idiopathic normal-pressure hydrocephalus. Sci Rep. 2019; 9(1): 16255, doi: 10.1038/s41598-019-52448-3, indexed in Pubmed: 31700018.

58. Souza RK, Rocha SF, Martins RT, et al. Gait in normal pressure hydrocephalus: characteristics and effects of the CSF tap test. Arq Neuropsiquiatr. 2018; 76(5): 324-331, doi: 10.1590/0004282X20180037, indexed in Pubmed: 29898079.

59. Kuba $\mathrm{H}$, Inamura T, Ikezaki K, et al. Gait disturbance in patients with low pressure hydrocephalus. J Clin Neurosci. 2002; 9(1): 33-36, doi: 10.1054/jocn.2001.1010, indexed in Pubmed: 11749014.

60. Huang X, Mahoney JM, Lewis MM, et al. Both coordination and symmetry of arm swing are reduced in Parkinson's disease. Gait Posture.
2012; 35(3): 373-377, doi: 10.1016/j.gaitpost.2011.10.180, indexed in Pubmed: 22098825.

61. Prakash KM, Ting SKS, Ahmad MT. Unusually increased arm swing in an apraxic upper limb in corticobasal syndrome. Parkinsonism Relat Disord. 2011; 17(2): 126-127, doi: 10.1016/j.parkreldis.2010.09.008, indexed in Pubmed: 20971029.

62. Williams DR, de Silva R, Paviour DC, et al. Characteristics of two distinct clinical phenotypes in pathologically proven progressive supranuclear palsy: Richardson's syndrome and PSP-parkinsonism. Brain. 2005; 128(Pt 6): 1247-1258, doi: 10.1093/brain/awh488, indexed in Pubmed: 15788542.

63. Zijlmans J. The L-dopa response in vascular parkinsonism. J Neurol Neurosurg Psychiatry. 2004; 75(4): 545-547, doi: 10.1136/ jnnp.2003.018309, indexed in Pubmed: 15026493.

64. Charlett A, Weller C, Purkiss AG, et al. Breadth of base whilst walking: effect of ageing and parkinsonism. Age Ageing. 1998; 27(1): 49-54, doi: 10.1093/ageing/27.1.49, indexed in Pubmed: 9504366.

65. Bovonsunthonchai S, Vachalathiti R, Pisarnpong A, et al. Spatiotemporal gait parameters for patients with Parkinson's disease compared with normal individuals. Physiother Res Int. 2014; 19(3): 158-165, doi: 10.1002/pri.1579, indexed in Pubmed: 24375990.

66. Abdo WF, Borm GF, Munneke M, et al. Ten steps to identify atypical parkinsonism. J Neurol Neurosurg Psychiatry. 2006; 77(12): 1367-1369, doi: 10.1136/jnnp.2006.091322, indexed in Pubmed: 16847047.

67. Stolze H, Kuhtz-Buschbeck JP, Drücke H, et al. Comparative analysis of the gait disorder of normal pressure hydrocephalus and Parkinson's disease. J Neurol Neurosurg Psychiatry. 2001; 70(3): 289-297, doi: 10.1136/jnnp.70.3.289, indexed in Pubmed: 11181848.

68. Damasceno BP, Carelli EF, Honorato DC, et al. The predictive value of cerebrospinal fluid tap-test in normal pressure hydrocephalus. Arq Neuropsiquiatr. 1997; 55(2): 179-185, doi: 10.1590/s0004-282x1997000200003, indexed in Pubmed: 9629375.

69. Zetusky WJ, Jankovic J, Pirozzolo FJ. The heterogeneity of Parkinson's disease: clinical and prognostic implications. Neurology. 1985; 35(4): 522-526, doi: 10.1212/wnl.35.4.522, indexed in Pubmed: 3982637.

70. Jankovic J, McDermott M, Carter J, et al. Variable expression of Parkinson's disease: a base-line analysis of the DATATOP cohort. The Parkinson Study Group. Neurology. 1990; 40(10): 1529-1534, doi: 10.1212/wnl.40.10.1529, indexed in Pubmed: 2215943.

71. Munhoz RP, Li JY, Kurtinecz M, et al. Evaluation of the pull test technique in assessing postural instability in Parkinson's disease. Neurology. 2004; 62(1): 125-127, doi: 10.1212/wnl.62.1.125, indexed in Pubmed: 14718714.

72. Hausdorff JM. Gait dynamics in Parkinson's disease: common and distinct behavior among stride length, gait variability, and fractal-like scaling. Chaos. 2009; 19(2): 026113, doi: 10.1063/1.3147408, indexed in Pubmed: 19566273.

73. Chee R, Murphy A, Danoudis M, et al. Gait freezing in Parkinson's disease and the stride length sequence effect interaction. Brain. 2009; 132(Pt 8): 2151-2160, doi: 10.1093/brain/awp053, indexed in Pubmed: 19433440.

74. Hely MA, Reid WGJ, Adena MA, et al. The Sydney multicenter study of Parkinson's disease: the inevitability of dementia at 20 years. Mov Disord. 2008; 23(6): 837-844, doi: 10.1002/mds.21956, indexed in Pubmed: 18307261.

75. Tan DM, McGinley JL, Danoudis ME, et al. Freezing of gait and activity limitations in people with Parkinson's disease. Arch Phys Med Rehabil. 2011; 92(7): 1159-1165, doi: 10.1016/j.apmr.2011.02.003, indexed in Pubmed: 21704798. 
76. Giladi N, McDermott MP, Fahn S, et al. Parkinson Study Group. Freezing of gait in PD: prospective assessment in the DATATOP cohort. Neurology. 2001; 56(12): 1712-1721, doi: 10.1212/wnl.56.12.1712, indexed in Pubmed: 11425939.

77. Osaki Y, Morita Y, Miyamoto Y, et al. Freezing of gait is an early clinical feature of progressive supranuclear palsy. Neurol Clin Neurosci. 2017; 5(3): 86-90, doi: 10.1111/ncn3.12122, indexed in Pubmed: 28702192.

78. Factor SA. The clinical spectrum of freezing of gait in atypical parkinsonism. Mov Disord. 2008; 23(Suppl 2): S431-S438, doi: 10.1002/ mds.21849, indexed in Pubmed: 18668624.

79. Gurevich T, Giladi N. Freezing of gait in multiple system atrophy (MSA). Parkinsonism Relat Disord. 2003; 9(3): 169-174, doi: 10.1016/s13538020(02)00049-4, indexed in Pubmed: 12573873.

80. Williams DR, Holton JL, Strand K, et al. Pure akinesia with gait freezing: a third clinical phenotype of progressive supranuclear palsy. Mov Disord. 2007; 22(15): 2235-2241, doi: 10.1002/mds.21698, indexed in Pubmed: 17712855.

81. Factor SA, Higgins DS, Qian J. Primary progressive freezing gait: a syndrome with many causes. Neurology. 2006; 66(3): 411-414, doi: 10.1212/01.wnl.0000196469.52995.ab, indexed in Pubmed: 16476942.

82. Spildooren J, Vercruysse S, Desloovere K, et al. Freezing of gait in Parkinson's disease: the impact of dual-tasking and turning. Mov Disord. 2010; 25(15): 2563-2570, doi: 10.1002/mds.23327, indexed in Pubmed: 20632376.

83. Espay AJ, Fasano A, van Nuenen BFL, et al. "On" state freezing of gait in Parkinson disease: a paradoxical levodopa-induced complication. Neurology. 2012; 78(7): 454-457, doi: 10.1212/WNL.0b013e3182477ec0, indexed in Pubmed: 22262741.

84. Bartels AL, Balash Y, Gurevich T, et al. Relationship between freezing of gait (FOG) and other features of Parkinson's: FOG is not correlated with bradykinesia. J Clin Neurosci. 2003; 10(5): 584-588, doi: 10.1016/ s0967-5868(03)00192-9, indexed in Pubmed: 12948464.

85. Peterson DS, Plotnik M, Hausdorff JM, et al. Evidence for a relationship between bilateral coordination during complex gait tasks and freezing of gait in Parkinson's disease. Parkinsonism Relat Disord. 2012; 18(9): 1022-1026, doi: 10.1016/j.parkreldis.2012.05.019, indexed in Pubmed: 22717367.

86. Bhatt H, Pieruccini-Faria F, Almeida QJ. Dynamics of turning sharpness influences freezing of gait in Parkinson's disease. Parkinsonism Relat Disord. 2013; 19(2): 181-185, doi: 10.1016/j.parkreldis.2012.09.006, indexed in Pubmed: 23083513.

87. Spildooren J, Vercruysse S, Meyns $P$, et al. Turning and unilateral cueing in Parkinson's disease patients with and without freezing of gait. Neuroscience. 2012; 207: 298-306, doi: 10.1016/j.neuroscience.2012.01.024, indexed in Pubmed: 22285883.

88. Plotnik M, Giladi N, Hausdorff JM. Is freezing of gait in Parkinson's disease a result of multiple gait impairments? Implications for treatment. Parkinsons Dis. 2012; 2012: 459321, doi: 10.1155/2012/459321, indexed in Pubmed: 22288021.

89. Lewis SJG, Barker RA. A pathophysiological model of freezing of gait in Parkinson's disease. Parkinsonism Relat Disord. 2009; 15(5): 333-338, doi: 10.1016/j.parkreldis.2008.08.006, indexed in Pubmed: 18930430.

90. DeLong MR, Wichmann T. Circuits and circuit disorders of the basal ganglia. Arch Neurol. 2007; 64(1): 20-24, doi: 10.1001/archneur.64.1.20, indexed in Pubmed: 17210805.

91. Vandenbossche J, Deroost N, Soetens E, et al. Freezing of gait in Parkinson's disease: disturbances in automaticity and control. Front Hum Neurosci. 2012; 6: 356, doi: 10.3389/fnhum.2012.00356, indexed in Pubmed: 23335895.
92. Heremans E, Nieuwboer A, Spildooren J, et al. Cognitive aspects of freezing of gait in Parkinson's disease: a challenge for rehabilitation. J Neural Transm (Vienna). 2013; 120(4): 543-557, doi: 10.1007/s00702-0120964-y, indexed in Pubmed: 23328947.

93. Jacobs JV, Nutt JG, Carlson-Kuhta P, et al. Knee trembling during freezing of gait represents multiple anticipatory postural adjustments. Exp Neurol. 2009; 215(2): 334-341, doi: 10.1016/j.expneurol.2008.10.019, indexed in Pubmed: 19061889.

94. Nieuwboer A, Dom R, De Weerdt W, et al. Electromyographic profiles of gait prior to onset of freezing episodes in patients with Parkinson's disease. Brain. 2004; 127(Pt 7): 1650-1660, doi: 10.1093/brain/ awh189, indexed in Pubmed: 15128621.

95. Silva FR, Muniz AM, Cerqueira LS, et al. Biomechanical alterations of gait on overweight subjects. Research on Biomedical Engineering. 2018; 34(4): 291-298, doi: 10.1590/2446-4740.180017.

96. Höglinger GU, Respondek G, Stamelou M, et al. Movement Disorder Society-endorsed PSP Study Group. Clinical diagnosis of progressive supranuclear palsy: The movement disorder society criteria. Mov Disord. 2017; 32(6): 853-864, doi: 10.1002/mds.26987, indexed in Pubmed: 28467028.

97. Scott G, Menz HB, Newcombe L. Age-related differences in foot structure and function. Gait Posture. 2007; 26(1): 68-75, doi: 10.1016/j. gaitpost.2006.07.009, indexed in Pubmed: 16945538.

98. Amboni M, Barone P, Hausdorff JM. Cognitive contributions to gait and falls: evidence and implications. Mov Disord. 2013; 28(11): 1520-1533, doi: 10.1002/mds.25674, indexed in Pubmed: 24132840.

99. Wilson J, Yarnall AJ, Craig CE, et al. Cholinergic basal forebrain volumes predict gait decline in Parkinson's disease. Mov Disord. 2021; 36(3): 611-621, doi: 10.1002/mds.28453, indexed in Pubmed: 33382126.

100. Thevathasan W, Debu B, Aziz T, et al. Movement Disorders Society PPN DBS Working Groupin collaboration with the World Society for Stereotactic and Functional Neurosurgery. Pedunculopontine nucleus deep brain stimulation in Parkinson's disease: a clinical review. Mov Disord. 2018; 33(1): 10-20, doi: 10.1002/mds.27098, indexed in Pubmed: 28960543.

101. Hoops S, Nazem S, Siderowf AD, et al. Validity of the MoCA and MMSE in the detection of $\mathrm{MCl}$ and dementia in Parkinson disease. Neurology. 2009; 73(21): 1738-1745, doi: 10.1212/WNL.0b013e3181c34b47, indexed in Pubmed: 19933974.

102. Leinonen V, Koivisto AM, Savolainen S, et al. Amyloid and tau proteins in cortical brain biopsy and Alzheimer's disease. Ann Neurol. 2010; 68(4): 446-453, doi: 10.1002/ana.22100, indexed in Pubmed: 20976765.

103. Kojima G, Masud T, Kendrick D, et al. Does the timed up and go test predict future falls among British community-dwelling older people? Prospective cohort study nested within a randomised controlled trial. BMC Geriatr. 2015; 15: 38, doi: 10.1186/s12877-015-0039-7, indexed in Pubmed: 25887660.

104. Pilloni G, Pau M, Costici F, et al. Use of 3D gait analysis as predictor of Achilles tendon lengthening surgery outcomes in children with cerebral palsy. Eur J Phys Rehabil Med. 2019; 55(2): 250-257, doi: 10.23736/ S1973-9087.18.05326-1, indexed in Pubmed: 30156089.

105. Mc Ardle R, Galna B, Donaghy P, et al. Do Alzheimer's and Lewy body disease have discrete pathological signatures of gait? Alzheimers Dement. 2019; 15(10): 1367-1377, doi: 10.1016/j.jalz.2019.06.4953, indexed in Pubmed: 31548122.

106. Mc Ardle R, Del Din S, Galna B, et al. Differentiating dementia disease subtypes with gait analysis: feasibility of wearable sensors? Gait Posture. 2020; 76: 372-376, doi: 10.1016/j.gaitpost.2019.12.028, indexed in Pubmed: 31901765.

107. Sim I. Mobile devices and health. N EngI J Med. 2019; 381(10): 956-968, doi: 10.1056/NEJMra1806949, indexed in Pubmed: 31483966. 